\title{
Dicke Bilder durch dünne Leitungen Konzepte und Algorithmen der Bilddatenkompression
}

\author{
von Ruedi Seiler und Klaus Jung
}

\begin{abstract}
Die meisten von uns kennen den Frust, vor dem Bildschirm zu sitzen und zu warten, bis endlich ein Bild stückweise und ruckartig zu erscheinen beliebt. Bilddaten sind so riesig, dass sie auch gute Datenkanäle zustopfen. Dies zu verhindern oder mindestens deutlich einzugrenzen, führt zu mathematisch interessanten Modellierungsund Optimierungsproblemen. Dabei spielen Konzepte aus der mathematischen Physik und der Informationstheorie eine zentrale Rolle, ganz besonders das Konzept der Entropie.
\end{abstract}

Spätere Generationen werden über unsere heutigen Ansätze lachen, Bilddaten zu bearbeiten und zu komprimieren, weil sie ganz offensichtlich am tatsächlichen Problem vorbeigehen. Die biologischen Systeme sind uns da ein gutes Stück voraus. Obwohl mehr die Auswertung als die Kompression die Spezialität unseres Sehapparates zu sein scheint, ist offenbar unser Auge dennoch zu einer beachtlichen Kompression fähig. ${ }^{1}$ Trotz enormer Anstrengungen ist jedoch die menschliche Wahrnehmung noch immer nicht genügend gut verstanden, um als Wegweiser einer guten Komprimierung dienen zu können. Sicherlich versuchen die neuen MPEG-Standards einen Schritt in diese Richtung zu gehen, scheitern jedoch in der Praxis an der Komplexität der dazu konzipierten Algorithmen.

Die heutigen Kompressionsalgorithmen tun, was in der Physik seit je die gängige Strategie war: Man sortiert die großen Datenmengen nach Skalen mit dem Ziel, die irrelevanten auszusortieren. Der relevante Anteil ist dann aber immer noch sehr umfangreich; diese Daten werden deshalb in einem zweiten Schritt statistisch analysiert, modelliert und und dann einem sogenannten Entropie-Kodierer zugeführt.

Zum Sortieren der Bilddaten werden hauptsächlich zwei Verfahren benutzt: Die diskrete Cosinustransformation (DCT) - wie sie etwa im populären „JPEG“Verfahren Verwendung findet - sowie die diskrete Wavelet-Transformation (DWT). Der neue Standard JPEG 2000 verwendet eine DWT. Er sortiert nach Frequenzen bzw. Längenskalen im Bild. Der DWT verwandt ist die Methode der fraktalen Kodierung, die zwar mathematisch besonders interessant ist, jedoch keine praktische Relevanz besitzt.

Die gängigsten Entropie-Kodierer sind der Huffmann Kodierer und der Arithmetische Kodierer. Sie funktionieren beide nach dem ENRISTUDA-Prinzip: Es wird ein statistisches Modell zur Modellierung der auftretenden Daten definiert, z. B. über die Messung der Häufigkeit der auftretenden Buchstaben. In einem indogermanischen Text tritt der Buchstabe E am häufigsten auf, danach kommt $\mathrm{N}$ und dann $\mathrm{R}$. E wird nun mit dem kürzesten Kodewort übertragen, N mit dem zweitkürzesten. Im Arithmetischen Kodierer wird die gesamte Botschaft in ein einziges Intervall kodiert, das dann mit einer hohen Präzision übertragen werden muss. Dies stellt sich schematisch so dar:

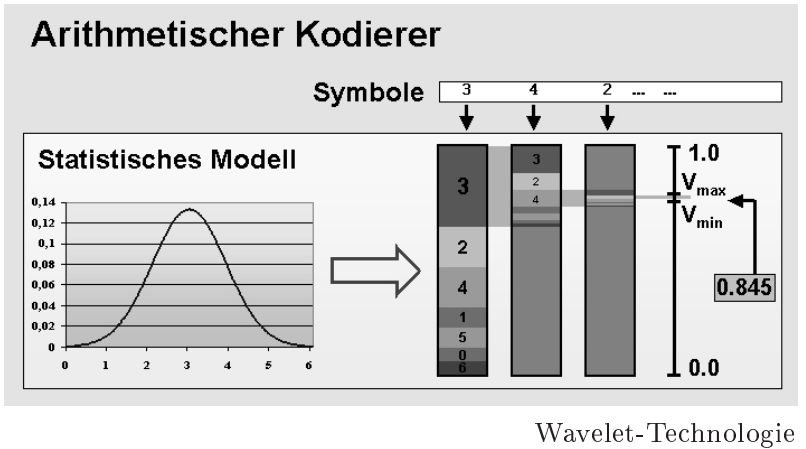

Diese Methode unterscheidet sich im Ansatz deutlich vom Lempel-Ziv Kode (LZ), der nicht zur Klasse der Entropiekodierer ${ }^{2}$ gehört. Alle drei sind für sehr große Datenmengen optimal, d.h. sie saturieren die von Shannon gefundene untere Schranke an die Länge des Kodes. Für Datenmengen wie sie in der Praxis der Bilddatenkompression auftreten sind die genannten Entropiekodierer besser geeignet als der LZ-Kode.

Die interessantesten mathematischen Fragen im Kontext der Bilddatenkompression kommen aus dem Anliegen, den Kode schnell zu machen und die Qualität der komprimierten Bilder bei vorgegebener Filegröße zu optimieren. Zwei davon sollen kurz erwähnt werden, die Faktorisierung von Wavelets und die rate allocation.

\footnotetext{
1 Eine grobe Abschätzung ergibt, dass die auf der Netzhaut ankommenden Signale ungefähr hundertmal komprimiert werden, bevor die darin enthaltene Information über den Sehnerv zum Hirn geschickt wird. Die Zahl der Photorezeptoren ist etwa $10^{8}$ und damit ungefähr 100 mal größer als die Zahl der Fasern im optischen Nerv. Er hat eine Bandbreite von ca. 5 MByte pro Sekunde. 2 Entropiekodierer benützen typischerweise ein Histogramm der vorkommenden Symbole.
} 
Kompressionsalgorithmen, die auf der Wavelet-Technologie aufsetzen, waren lange Zeit durch die hohe Komplexität behindert. Dies änderte sich in den letzten Jahren zum einen durch die Verfügbarkeit schnellerer Prozessoren, und zum anderen auf Grund einer algorithmischen Verbesserung, dem lifting. Sie kann am besten als Faktorisierung von Schleifen in einer Matrixgruppe verstanden werden, hierzu später mehr.

Die rate allocation ist eines der interessantesten Probleme der Informationstheorie, das eine Fülle mathematischer Aspekte enthält. Für den Fall, dass die Daten durch eine Gaußsche Quelle modelliert werden, führt es auf ein Kugelpackungsproblem.

Eine immer bessere Kompression von Bilddaten ist trotz der schnell zunehmenden Bandbreiten der Datennetze - von großer praktischer Relevanz. Dafür gibt es mehrere Gründe: Erstens steigt die Menge der ausgetauschten Daten exponentiell mit der Zahl der Nutzer, und damit werden die Netzanbieter Mühe haben, die gegenwärtige Situation qualitativ zu verbessern. Zweitens gibt es Datenkanäle, deren Bandbreite aus technischen, physikalischen oder Kostengründen eingeschränkt sind, wie zum Beispiel im mobilen Bereich oder bei Verbindungen über Satelliten. Hier lohnt sich der Einsatz guter Kompressionstechnologie ganz besonders; denn die jährliche Miete eines Transponders von Astra mit einer Bandbreite von 2 MByte pro Sekunde beträgt ungefähr 1,2 Millionen DM pro Jahr. Wenn beispielsweise über einen dieser Kanäle nicht nur ein Programm, sondern zwei oder gar drei Programme ausgestrahlt werden können, ist dies ein beachtlicher Erfolg.

\section{Kompressionsalgorithmen}

Ein Bild wird digital durch eine Anzahl von Bildpunkten mit Farb- und Helligkeitswerten dargestellt. Als Farben werden meist rot, grün und blau oder eine Kombination davon gewählt, z.B. die Gesamtintensität und die beiden Farbtöne Blau und Rot. Ein einzelner Bildpunkt hat auf diese Weise einen dreidimensionalen Zustandsraum. Die Intensitätswerte der einzelnen Farben im RGB-Raum werden meistens mit 8 Bits dargestellt, womit dann ein digitales Bild von 1000 Pixeln Breite und 800 Pixel Höhe eine Filegröße von 2,4 MByte besitzt. Im Vergleich zu einer Textseite vergleichbarer Dimension ist dies ungefähr 1000 mal mehr! Um eine Videosequenz mit 25 Bildern pro Sekunde zu übertragen, wie dies im Fernsehen üblich ist, wäre eine Bandbreite von 65 MByte pro Sekunde nötig. Über den gleichen Datenkanal könnten hingegen 60.000 Handys betrieben werden, denn diese müssen zur Zeit noch mit gut einem Kilobyte pro Sekunde Bandbreite auskommen.

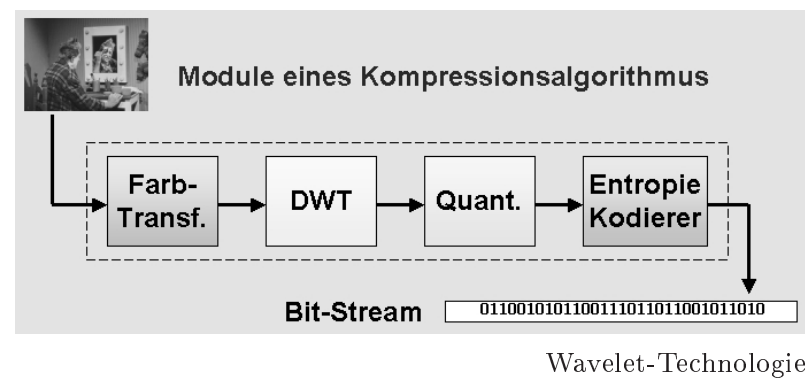

So gesehen schiene das Problem der Bildübertragung fast hoffnungslos, gäbe es nicht zwei Mechanismen unseres Sehapparates, die uns entgegenkommen. Der erste ist die unwillkürliche Zerlegung eines Bildes in bekannte Objekte, der zweite die Unempfindlichkeit des Auges gegenüber einer großen Zahl von Bildveränderungen, wie zum Beispiel kleinen lokalen Verzerrungen und geringfügigen Verschiebungen. So ist es empirisch erwiesen, dass Bildanteile mit kleinen Längenskalen von unserem Auge kaum wahrgenommen werden.

Die Zerlegung eines Bildes in bekannte, meist homogene Teile ist das Anliegen der zweiten Generation von Kompressionsalgorithmen. Eine erfolgreiche Implementierung, d.h. eine schnelle Segmentierung eines natürlichen Bildes in homogene Anteile, kann gegenwärtig jedoch noch nicht in vernünftiger Zeit durchgeführt werden.

Die zur Zeit besten Kompressionsalgorithmen gehören deshalb alle noch zur ersten Generation, die allein vom zweiten Mechanismus Gebrauch machen. Sie sortieren die kleinen Längenskalen aus und werfen sie ganz oder teilweise weg $-\mathrm{d}$. h. führen eine sogenannte Quantisierung durch. Für Videokodes wird dazu eine diskrete Fouriertransformation (DCT) verwendet, für Einzelbilder setzen sich mehr und mehr diskrete Wavelettransformationen durch.

Ein Kompressionsalgorithmus der ersten Generation für Einzelbilder ist schematisch folgendermaßen aufgebaut: Ein erstes Modul transformiert die drei Farben rot, grün und blau der Rohdaten in drei neue, meistens in die Intensität und zwei Farbanteile. Dies ist bereits ein erstes Sortieren nach wichtigen und unwichtigen Anteilen, denn das Auge ist gegenüber Intensitätsschwankungen ungleich emfindlicher als gegenüber kleinen Farbänderungen. Dieser Kunstgriff findet bereits seit einem halben Jahrhundert beim Farbfernsehen Anwendung.

Danach wird das Bild durch eine diskrete Wavelettransformation geschickt und so in drei Anteile mit den feinsten Längenskalen in horizontaler, vertikaler und horizontal-vertikaler Richtung vom Rest getrennt. Der Rest, der sogenannte „Tiefpass", ist ein Bild, das etwas verschwommen aussieht, weil ihm 
die Feinheiten fehlen. Er kann erneut durch den Wavelettransformer geschickt werden, wobei dann die nächstgröberen Skalen aussortiert werden. Die Bildanteile mit den feinen Skalen, die sogenannten „Hochpässe“, sind keine natürlichen Bilder. Sie enthalten nur bestimmte Elemente wie z. B. die vertikalen Streifen in der Tapete im nachfolgenden Bild.

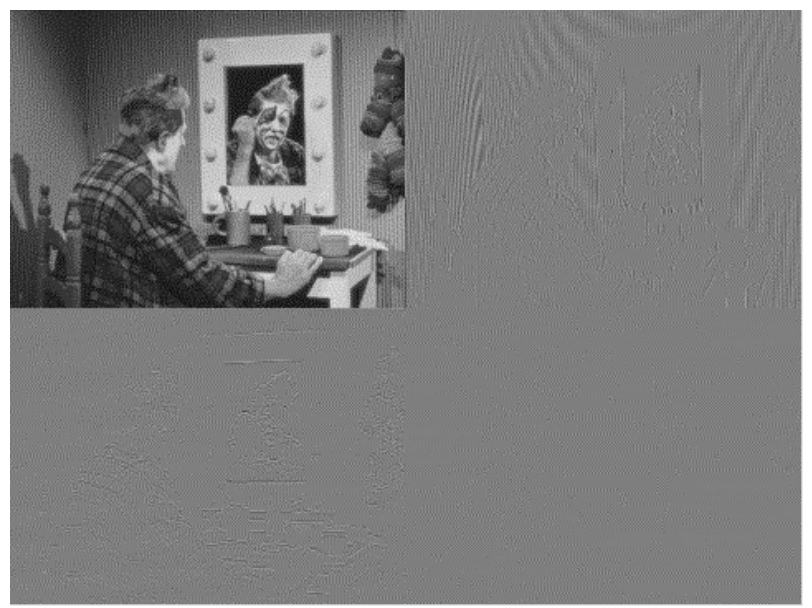

DWT-transformiertes Bild

Das nächste Modul ist der Quantisierer. Hier werden die Bilddaten zum ersten und einzigen Mal irreversibel verändert. Die Bilddaten der Hochpässe werden teils ganz weggelassen oder durch Treppenfunktionen mit nur wenigen Werten approximiert. Der Quantisierer wird entweder fest eingestellt oder durch die „Rate Allocation“ gesteuert. Sie optimiert die Qualität der Bilder bei vorgegebener Filegröße.

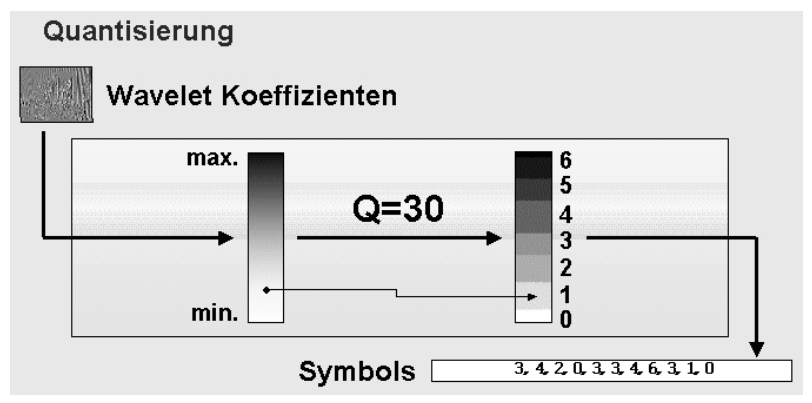

Wavelet-Technologie

Das letzte Modul ist der Entropie-Kodierer und ist typischerweise ein arithmetischer Kodierer. Das Grundprinzip ist bereits in der Einleitung beschrieben worden: Es wird in jedem der Hochpässe und im Tiefpass ein statistisches Modell ausgewählt, nach dem dann die Daten kodiert werden.

In dieser Skizze eines Kompressionsalgorithmus der ersten Generation haben wir geflissentlich die großen Probleme umschifft. Dazu gehört etwa die Frage, wie die Qualität eines komprimierten Bildes qualitativ erfasst werden kann, oder auch ,Was ist ein natürliches
Bild?" Auf beide Fragen gibt es nur unzureichende Antworten.

In diesem Zusammenhang stellt sich die Frage, um wieviel das menschliche Auge „betrogen“ werden kann, ohne dies zu bemerken. Diese Frage ist zugegebenermaßen nicht leicht $\mathrm{zu}$ beantworten, allein schon deshalb, weil sie nicht genau gefasst ist. Qualitativ kann hierzu folgendes gesagt werden: Natürliche Bilder, das sind in diesem Fall eine Reihe von Testbildern, wie „Lena“ oder „Barbara“, können mit einem Kodierer der ersten Generation etwas besser als $2: 1$ verlustfrei komprimiert werden. Mit Quantisierung, d.h. mit Verlust, bei visueller ausgezeichneter Qualität kann auf $20: 1$ oder noch höher komprimiert werden. Das menschliche Auge benötigt weniger als $10 \%$ der ursprünglich in den Daten vorhandenen Information, um ein perfektes Bild zu sehen.

Im folgenden werden kurz zwei mathematische Fragen beschrieben. Die erste betrifft die Geschwindigkeitsoptimierung des diskreten Wavelettransformers, die zweite die optimale Quantisierung.

\section{Faktorisierung diskreter Wavelettransformationen}

Vor vier Jahren haben W. Sweldons und I. Daubechies [2] bemerkt, dass diskrete Wavelettransformationen durch Faktorisierung um das doppelte beschleunigt werden können. Dieses Verfahren, das dem ersten Schritt einer schnellen Fouriertransformation ähnlich ist, kann am transparentesten als Faktorisierung von Schleifen in der Gruppe der invertierbaren $2 \times 2$ Matrizen $\mathrm{GL}(2, \mathbb{C})$ aufgefasst werden [5], [3].

Um dies und den Begriff des diskreten Wavelets etwas genauer zu erläutern, betrachten wir den Hilbertraum $\ell^{2}(\mathbb{Z})$ der quadratsummierbaren Folgen und den darauf definierten Shiftoperator $S,(S \Psi)_{n}:=$ $\Psi_{n-1}$. Zu jedem diskreten Wavelet $g \in \ell^{2}(\mathbb{Z})$ gehört eine diskrete Skalenfunktion $h \in \ell^{2}(\mathbb{Z})$. Dieses Paar erzeugt zusammen mit dem Quadrat des Shifts eine Basis in $\ell^{2}(\mathbb{Z})$ : Das einfachste Beispiel eines solchen Paares ist das diskrete Haar-Wavelet $g_{0}:=\left(\delta_{0,}-\delta_{1,}\right) / \sqrt{2}$ zusammen mit der diskreten Haar-Skalenfunktion $h_{0}:=\left(\delta_{0, \cdot}+\delta_{1}, \cdot\right) / \sqrt{2}$.

Zur Zerlegung einer Folge $f \in \ell^{2}(\mathbb{Z})$ in Komponenten bezüglich der Basis $\left\{S^{n} h_{0}, S^{n} g_{0} \mid n \in 2 \mathbb{Z}\right\}$ müssen die Skalarprodukte $\left\{\left(h_{0}(\cdot-2 n), f(\cdot)\right)\right\}_{n \in \mathbb{Z}}$ und $\left\{\left(g_{0}(\cdot-2 n), f(\cdot)\right)\right\}_{n \in \mathbb{Z}}$ ausgerechnet werden. Die erste Koordinatengruppe entspricht einer Mittelung über die benachbarten Gitterpunkte $\{2 n, 2 n+1\}$, die zweite der Differenz der Werte $f(2 n)$ und $f(2 n+1)$ und somit einer diskreten Ableitung. Damit wird $f$ in einen ,Tiefpass" und in einen ,Hochpass“ zerlegt. 


$$
\begin{array}{cc}
\left(\begin{array}{cc}
\frac{1}{8}\left(z^{-1}+6+z\right) & \frac{1}{32}\left(-3 z^{-2}-5 z^{-1}-5-3 z\right) \\
\frac{1}{2}(1+z) & \frac{1}{8}\left(-3 z^{-1}+10-3 z\right)
\end{array}\right)= \\
\left(\begin{array}{cc}
1 & \frac{1}{4} \\
0 & 1
\end{array}\right)\left(\begin{array}{cc}
1 & \frac{1}{4} z^{-1} \\
0 & 1
\end{array}\right)\left(\begin{array}{cc}
1 & 0 \\
z & 1
\end{array}\right)\left(\begin{array}{ll}
1 & 0 \\
1 & 1
\end{array}\right)\left(\begin{array}{ccc}
1 & -\frac{3}{16} z^{-1} \\
0 & 1
\end{array}\right)\left(\begin{array}{cc}
\frac{1}{2} & -\frac{3}{8} \\
0 & 2
\end{array}\right)
\end{array}
$$

Eine diskrete Skalenfunktion $h$ und das zugehörige Wavelet $g$ können durch diejenige lineare invertierbare Abbildung $T$ charakterisiert werden, die die Vektoren $\left\langle S^{n} g_{0}, S^{n} h_{0}\right\rangle$ auf $\left\langle S^{n} g, S^{n} h\right\rangle, n \in 2 \mathbb{Z}$, abbildet. $T$ vertauscht mit $S^{2}$ und kann deshalb zusammen mit $S^{2}$ diagonalisiert werden. Das Spektrum von $S^{2}$ ist offensichtlich der Kreis $|z|=1$; $T$ wirkt auf den Eigenraum von $S^{2}$ zum Eigenwert $z \in S^{1}$ als die invertierbare $2 \times 2$ Matrix $T(z)$. Falls $g$ und $h$ kompakten Träger haben, ist $T(z)$ ein Laurent-Polynom in $z$ mit Werten in $\operatorname{GL}(2, \mathbb{C})$, also eine polynominiale Schleife in der Matrixgruppe $\operatorname{GL}(2, \mathbb{C})$. Eine solche Schleife kann in elementare Faktoren zerlegt werden; oben ist ein Beispiel [3] wiedergegeben.

Es stellt den kubischen $B$-Spline in der Sprache der Schleifen in $G L(2, C)$ dar. Der Zerlegungsalgorithmus ist verhältnismäßig einfach und kann z. B. als Mathematica-Programm implementiert werden.

Die Reduktion der Komplexität auf etwas mehr als die Hälfte kann an diesem Beispiel leicht verifiziert werden.

Die Reduktion der arithmetischen Komplexität der DWT um einen Faktor zwei mag diejenigen unter uns wenig beeindrucken, die sich mit den JahrtausendFragen $\mathcal{P}$ gleich $\mathcal{N} \mathcal{P}$ oder $\mathcal{P}$ nicht gleich $\mathcal{N} \mathcal{P}$ befassen. Für die praktische Anwendung im Bereich der Bilddatenkompression ist dieser kleine Erfolg jedoch von großer Bedeutung, denn in die arithmetische Komplexität des Kompressionsalgorithmus geht das Produkt der Komplexität der DWT multipliziert mit der Anzahl der Bildpunkte ein, und diese ist sehr groß.

\section{Optimale Quantisierung}

Auf den ersten Blick scheint die Aufgabe des Quantisierers einfach zu sein: Er soll die wavelettransformierten Bilddaten durch Treppenfunktionen mit nur wenigen Werten approximieren. Diese Aufgabe optimal zu lösen ist jedoch erstaunlich komplex und kann nur in einem sehr eingeschränkten Rahmen gelöst werden.

Etwas genauer gefasst wird dem Quantisierer eine Kompressionsrate zugewiesen, und er soll dann die Approximation des Bildes mit der geringsten Verzerrung finden. Ein Maß der Verzerrung, das unseren visuellen Eindruck gut modelliert, gibt es jedoch nicht.
Das beste ist es, den normierten $L^{2}$-Abstand von Bild und komprimiertem Bild zu nehmen.

Überhaupt eine vernünftige Kompressionsrate erzielen zu können, scheint auf den ersten Blick ein hoffnungsloses Unterfangen, denn die von der diskreten Wavelettransformation erzeugten Koeffizienten haben typischerweise mehr als dreißig binäre Stellen das bedeutet, dass die transformierten Daten ungefähr vier mal größer sind als das ursprüngliche Bild. Dass hier dennoch eine Quantisierung gefunden werden kann, die der nachfolgende Entropiekodierer so stark zusammenstauchen kann, dass am Ende eine gute Kompression herauskommt, hängt im wesentlichen an zwei Dingen: Erstens kann die Kompressionsrate nach der Shannonschen Theorie als die Entropie eines statistischen Modells gedeutet werden und zweitens gibt es für die wavelettransformierten Daten von natürlichen Bildern ein gutes statistisches Modell.

Bereits in der Einleitung wurde das ENRISTUDAPrinzip zur Kompression von indogermanischen Texten angesprochen; es basiert auf einem statistischen Modell der auftretenden Daten. Es ist deshalb naheliegend, auch Bilddaten als typische Konfigurationen eines Zufallsprozesses aufzufassen. Dies ist aus der statistischen Mechanik von Spinsystemen wohl bekannt. Die Spinkonfigurationen sind nach dem Gibbsschen Maß verteilt und lassen sich in typische und untypische zerlegen. Die Anzahl der typischen Konfigurationen $\tau$ wächst im thermodynamischen Limes, d.h. für sehr große schwarz-weiß Bilder, exponentiell, $|\tau| \sim 2^{N H}$, wobei $N$ die Anzahl der Bildpunkte und $H$ die Entropie pro Bildpunkt bezeichnet. Die typischen Konfigurationen können somit durch binäre Zahlen mit NH Stellen durchnumeriert werden. $H$ ist deshalb die zur Kodierung der typischen Konfigurationen notwendige Anzahl der Bits pro Bildpunkt. Dies ist der Inhalt des Satzes von ShannonMcMillan-Breiman. Es ist die Aufgabe des Kodierermodules, die vom Quantisierer aufgearbeiteten Daten auf den Shannon-Limes zu komprimieren, d.h. die Kompressionsrate ist gleich eins durch die Entropie.

Nun geht es um ein gutes statistisches Modell für natürliche Bilder. Was natürliche Bilder sind, ist weitgehend unklar und ein interessantes Gebiet der Forschung. Simoncelli hat die Verteilung der Konfigurationen einzelner Bildpunkte in den Hochpässen unter- 
sucht und festgestellt, dass sie sehr gut durch verallgemeinerte Gaußfunktionen $p(x) \sim \exp \left(-\left|\frac{x}{s}\right|^{\alpha}\right)$ dargestellt werden können [1]. Mit diesem Ansatz unabhängig gleichverteilter Konfigurationen kann das Optimierungsproblem für die Familie der sogenannten „Equiquantisierer mit Deadzone“ gelöst werden, d.h. in dieser Familie von Quantisierern kann derjenige gefunden werden, für den die Verzerrung zwischen Original und komprimierten Bild - bei vorgegebener Kompressionsrate - am geringsten ist.

Dieses Problem kann auch geometrisch verstanden werden, wie dies in der nachfolgenden Skizze dargestellt ist.

Rate-Distortion $\mathrm{D}(\mathrm{R})$ als Kugelpackungsproblem

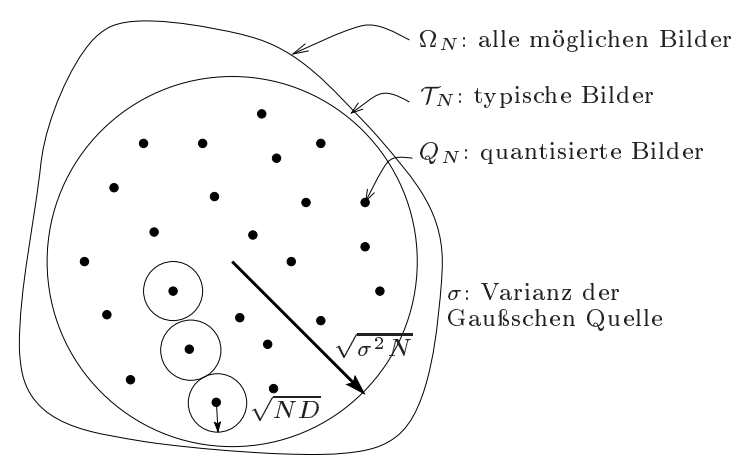

In der Menge aller möglichen Bilder $\Omega_{N}$ gibt es die Menge der typischen $\tau_{N}$. Diese sollen nun durch eine geschickte Auswahl $\mathcal{Q}_{N}$ approximativ dargestellt werden, d. h. jedes Element von $\tau$ muss in einer Kugel mit Radius $\sqrt{N D}$ enthalten sein; $N$ ist die Zahl der Bildpunkte und $D$ die Verzerrung. Dies ist das Kugelpackungsproblem, von dem im ersten Abschnitt die Rede war.

Die Familie der Equiquantisierer approximiert die Intensitäts- und Farbwerte der einzelnen Bildpunkte durch Treppenfunktionen derart, dass benachbarte Werte gleichen Abstand $\triangle$ haben. Für den Zusammenhang von $\triangle$ und die Verzerrung $D$ gibt es eine über 100 Jahre alte Formel $D=\frac{1}{12} \triangle^{2}[4]$. Zusammen mit dem Shannonschen Ausdruck ${ }^{3} D(R)=\sigma^{2} 2^{-2 R}$ für die beste Verzerrung $D$ bei vorgegebener Rate $R$ - hier für den Fall einer Gaußschen Quelle mit Varianz $\sigma^{2}-$ kann die Schrittweite $\triangle$ als Funktion von $R$ ausgedrückt werden und definiert einen Algorithmus für die optimale Quantisierung. Damit ist das Problem der „Rate Allcoation“ für die einfache Familie der Equiquantisierer mit Schrittweite $\triangle$ gelöst.

\section{Literaturverzeichnis}

[1] E. P. Simoncelli: Statistical Models for Images: Compression, Restauration and Synthesis. 31st Asilomar Conf. on Signals, Systems and Computers, 1997, 673-678.

[2] W. Sweldons: The Lifting Scheme: A Custom-Design Construction of Biorthogonal Wavelets, Applied and Computational Harmonic Analysis 3, 1996, 186-200.

[3] S. Borac und R. Seiler: Loop Group Factorization of Biorthogonal Wavelet Bases, Proceedings of the Schladming Winter School, 1999.

[4] W. F. Sheppard: On the calculations of the most probable values of frequency constants for data arranged according to equidistant divisions of a scale, Proc. London Math. Soc., 24, 1998, 353-380.

[5] M. Holschneider: Wavelets: An Analysis Tool, Oxford University Press, Oxford, 1995.

\section{Adresse der Autoren}

Prof. Dr. Ruedi Seiler

FB Mathematik

TU Berlin

10623 Berlin

seiler@math.tu-berlin.de

Dr. Klaus Jung

AlgoVision Mediatec

12247 Berlin

kjung@algovision. com

3 Diese Formel kann leicht aus der obigen Skizze abgeleitet werden: Die Anzahl der typischen Zustände ist einerseits $2^{N} R$ andererseits - bis auf irrelevante Termen - gleich dem Quotienten der Volumina der beiden Kugeln in $R^{N}$ mit Radius $\sqrt{\sigma^{2} N}$ und $\sqrt{N D}$. Die Anzahl der Bits, die zur Nummerierung notwendig ist, sei mit $N R$ bezeichnet 